\title{
Barriers to VCT despite 13 years of community-based awareness campaigns in a peri-urban township in northern Limpopo
}

\author{
Petra De Koker, Pierre Lefèvre, Francine Matthys, Patrick van der Stuyft, Wim Delva
}

To the Editor: An estimated 5.7 million people in South Africa live with HIV / AIDS. ${ }^{1}$ In 2008, it was estimated that $12.5 \%$ of the Limpopo population aged 15 - 49 was HIV-positive, while the national HIV prevalence estimate was $18.8 \%{ }^{2}$

Over the past 8 years, the South African government has supported prevention campaigns, expanded voluntary counselling and testing (VCT) sites, and increased the access to antiretroviral therapy (ART) to decrease the burden of the epidemic. ${ }^{3,4} \mathrm{VCT}$ was offered at $87 \%$ of primary health care facilities in Limpopo Province in 2003. ${ }^{5}$ By $2007,42 \%$ of the people in need of ART in South Africa had commenced it. ${ }^{4}$ Despite VCT's benefits and the increased number of testing sites, many South Africans remain untested for HIV for various reasons.

In Bela-Bela, the HIV / AIDS Prevention Group (HAPG) has been active since 1996, providing VCT and care and support to people with HIV/AIDS. Its activities include prevention campaigns, free VCT and ART, home-based care and orphan care. Each year, $39.4-42.2 \%$ of the HAPG VCT attendees test HIV-positive. In 2005, 254 HIV/AIDS prevention activities were organised, reaching 15570 participants (73\% women).

We studied motivations for, and barriers to, VCT uptake among black South Africans living in Bela-Bela, a peri-urban town with a population of 55844 and an unemployment rate of $21 \%{ }^{6}$

\section{Methods}

Eight gender-mixed focus group discussions (FGDs) totalling 86 people, of whom $58 \%$ were women, were conducted in 2005. Participants ranged in age from 15 to 35 years (median 21 years). Four FGDs comprised people who had undertaken VCT; participants of the other 4 had not experienced VCT. The questions, for tested participants, focused on HIV / AIDSrelated knowledge, experience with VCT, motivations for and

International Centre for Reproductive Health, Ghent University, Ghent, Belgium; and School of Public Health, University of the Western Cape, Bellville, South Africa Petra De Koker, MA

Department of Public Health, Epidemiology and Disease Control Unit, Institute of Tropical Medicine, Antwerp, Belgium

Pierre Lefèvre, $\mathrm{PhD}$

Francine Matthys, MD

Patrick van der Stuyft, MD, PhD

International Centre for Reproductive Health, Ghent University, Ghent, Belgium: and South African Centre for Epidemiological Modelling and Analysis, Stellenbosch University

Wim Delva, MD

Corresponding author: Petra De Koker (petra.dekoker@ugent.be) barriers to VCT uptake, facilitating factors to go for VCT, and the effects of VCT. Non-tested participants were asked about knowledge related to HIV / AIDS and VCT, and barriers and facilitating factors for VCT uptake. FGDs were tape-recorded and transcribed verbatim. The analysis was supported by QSR N6 software (QSR International Pty Ltd, Melbourne, Australia). Data were coded and analysed by 2 researchers. A coding book was progressively structured, based on the research questions and emerging themes. Qualitative matrices were built to identify major trends in the data. The results were shared with the HAPG director, and ensuing discussions provided additional information to contextualise and update the findings.

\section{Results}

The perceived core motivations for VCT uptake were having casual sex partners and not using condoms. Fear appeared to be both the main motivation for VCT uptake and the core barrier. Fear of the test result, which was related to risky sexual behaviour such as having multiple partners or unsafe sex, and fear of discrimination, were the most cited fears.

Despite many participants saying they could easily access information and had numerous opportunities to attend awareness campaigns, they commonly expressed the need for more information to overcome their fears, to make wellconsidered decisions towards VCT uptake, and to incorporate preventive sexual behaviours. Enhancing communication between partners and family members and in the community were reported as factors that would facilitate HIV testing. Participants also commonly emphasised that HIV/AIDS and VCT information campaigns should be directed to specific target groups, such as separate male and female groups, to help people to cope with difficulties in undertaking VCT. Some of these barriers, such as fear of discrimination, still exist, and the participants expressed unfulfilled needs.

\section{Discussion}

The demand for additional information expressed by participants in an environment where HAPG has conducted many campaigns for 13 years is surprising. Having attended a prevention campaign was never mentioned as a motivation to seek VCT. A possible explanation is that the campaigns did not meet the attendees' needs - the content of the campaigns may leave some questions unanswered, and underlying fears and uncertainties related to testing, counselling and risky behaviour might not be addressed. However, would more information effectively take away or reduce all the fears expressed? The fear of discrimination may decrease because there might be more openness about the disease, while other fears such as 'getting sick' may not be effectively addressed 
by information campaigns. Another possibility is that people attending the campaigns were unable to adequately transfer knowledge gained from the group level, where it is given, to applying it to themselves. This lack of 'internalisation' hinders communicating about HIV/AIDS and VCT, in partner relationships and in the community. The request for more information could be an excuse to transfer individual responsibilities to the HAPG, thus justifying not having to change behaviours or undertake VCT. People may also need behavioural and life skills to be motivated to undertake VCT.

Although South Africa has experienced political and public debate about the existence of HIV/AIDS, ${ }^{7}$ we found that the disease is recognised as a major threat in Bela-Bela. However, despite HIV / AIDS and VCT information campaigns, these interventions seem unable to effectively address the barriers to VCT. In 2005, the lack of internalisation of knowledge detracted from the uptake of VCT. To enhance social communication about HIV / AIDS and to increase the effects of prevention campaigns and the spill-over effects of VCT in the community, we recommended that factual information should be complemented by providing communication, behavioural and life skills.

Four years later, with increased access to ART, has the situation changed, and is the need for the targeted information and communication skills reported in 2005 still present? Recent discussions with people from HAPG indicate that the content and focus of awareness and information campaigns has not changed. However, since 2009, there have been monthly doorto-door campaigns and weekly road campaigns whereby staff members address people from street corners, providing them with information regarding VCT and HIV/AIDS-related services, including ART. The Department of Health's shift towards provider-initiated counselling and testing of pregnant women has also increased VCT among this group in Bela-Bela.
Despite these changes, more information is still requested. Increased access to ART seems not to be a sufficiently large incentive to undertake HIV testing nor to decrease discrimination.

The need to open the discussion and communication about HIV / AIDS, VCT and sexuality at partner, family, community and national levels is essential in any action to decrease fear and stigmatisation, to increase VCT uptake to maximise its secondary preventive effects and as an entry point for ART, and to seriously decrease the burden of the disease.

We gratefully acknowledge Cecile Manhaeve and the staff members, volunteers and counsellors of the Bela-Bela HIV/AIDS Prevention Group for their assistance and support. We also thank the study participants for their willingness to co-operate and for their openness. We pay tribute to Goedele De Coene for her input as research assistant and for her support.

\section{References}

1. UNAIDS. Report on the Global AIDS Epidemic: August 2008. UNAIDS/08.25E/jc1510E. Geneva: UNAIDS, 2008.

2. Actuarial Society of South Africa. AIDS Committee of Actuarial Society of South Africa. 2003 Model: ProvOutput, 2003. http://www.hst.org.za/healthstats/84/data (accessed 20 October
2009).

3. Birdsall K, Hajiyiannis H, Nkosi Z, Parker W. Voluntary Counselling and Testing in South Africa: Analysis of Calls to the National AIDS Helpline. Johannesburg: Center for AIDS Development, Research and Evaluation, 2004

4. UNAIDS. AIDS Epidemic Update: December 2007. UNAIDS/07.27E / JC1322E. Geneva UNAIDS/WHO, 2006. http://data.unaids.org/pub/Report/2008/jc1526_epibriefs_ssafrica en.pdf (accessed 23 October 2009).

5. Irlam J, Reagon G, Levin J. The National Primary Health Care Facilities Survey 2003. Durban: Health Systems Trust, The Equity Project and Department of Health, 2004. http:/ / www.hst. org.za/publications/617/ (accessed 21 October 2009).

6. Statistics South Africa. Community Survey 2007: Statistical Release Basic Results Municipalities / Statistics South Africa. Pretoria: Statistics South Africa, 2008. http://www.statssa.gov.za/ publications/P03011/P030112007.pdf (accessed 21 October 2009).

7. Fassin D. When Bodies Remember. Experience and Politics of Aids in South Africa. Berkeley, Calif.: University of California Press, 2008

Accepted 10 February 2010. 